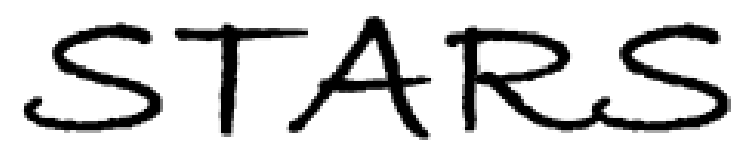

University of Central Florida

STARS

$1-1-2012$

\title{
Numerical Modeling of The 2009 Impact Event On Jupiter
}

\author{
Jarrad W. T. Pond \\ University of Central Florida \\ Csaba Palotai \\ University of Central Florida \\ Travis Gabriel \\ University of Central Florida \\ Donald G. Korycansky \\ Joseph Harrington \\ University of Central Florida
}

See next page for additional authors

Find similar works at: https://stars.library.ucf.edu/facultybib2010

University of Central Florida Libraries http://library.ucf.edu

This Article is brought to you for free and open access by the Faculty Bibliography at STARS. It has been accepted for inclusion in Faculty Bibliography 2010 s by an authorized administrator of STARS. For more information, please contactSTARS@ucf.edu.

\section{Recommended Citation}

Pond, Jarrad W. T.; Palotai, Csaba; Gabriel, Travis; Korycansky, Donald G.; Harrington, Joseph; and Rebeli, Noémi, "Numerical Modeling of The 2009 Impact Event On Jupiter" (2012). Faculty Bibliography 2010 s. 3152.

https://stars.library.ucf.edu/facultybib2010/3152

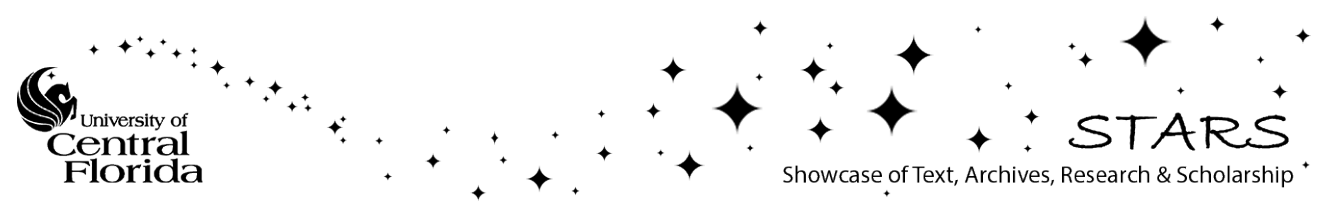




\section{Authors}

Jarrad W. T. Pond, Csaba Palotai, Travis Gabriel, Donald G. Korycansky, Joseph Harrington, and Noémi Rebeli 


\title{
NUMERICAL MODELING OF THE 2009 IMPACT EVENT ON JUPITER
}

\author{
Jarrad W. T. Pond ${ }^{1}$, Csaba Palotai ${ }^{1}$, Travis Gabriel ${ }^{1}$, Donald G. Korycansky ${ }^{2}$, \\ JOSEPH HARRINGTON ${ }^{1}$, AND NOÉMI REBELI ${ }^{1}$ \\ ${ }^{1}$ Planetary Sciences Group, Department of Physics, University of Central Florida, Orlando, FL 32816-2385, USA; jarradpond@ gmail.com \\ ${ }^{2}$ Department of Earth and Planetary Science, University of California, Santa Cruz, CA 95064, USA \\ Received 2011 November 2; accepted 2011 December 6; published 2012 January 6
}

\begin{abstract}
We have investigated the 2009 July impact event on Jupiter using the ZEUS-MP 2 three-dimensional hydrodynamics code. We studied the impact itself and the following plume development. Eight impactors were considered: $0.5 \mathrm{~km}$ and $1 \mathrm{~km}$ porous $\left(\rho=1.760 \mathrm{~g} \mathrm{~cm}^{-3}\right)$ and non-porous $\left(\rho=2.700 \mathrm{~g} \mathrm{~cm}^{-3}\right)$ basalt impactors, and $0.5 \mathrm{~km}$ and $1 \mathrm{~km}$ porous $\left(\rho=0.600 \mathrm{~g} \mathrm{~cm}^{-3}\right)$ and non-porous $\left(\rho=0.917 \mathrm{~g} \mathrm{~cm}^{-3}\right)$ ice impactors. The simulations consisted of these bolides colliding with Jupiter at an incident angle of $\theta=69^{\circ}$ from the vertical and with an impact velocity of $v=61.4 \mathrm{~km} \mathrm{~s}^{-1}$. Our simulations show the development of relatively larger, faster plumes created after impacts involving $1 \mathrm{~km}$ diameter bodies. Comparing simulations of the 2009 event with simulations of the Shoemaker-Levy 9 (SL9) events reveals a difference in plume development, with the higher incident angle of the 2009 impact leading to a shallower terminal depth and a smaller and slower plume. We also studied the amount of dynamical chaos present in the simulations conducted at the 2009 incident angle. Compared to the chaos of the SL9 simulations, where $\theta \approx 45^{\circ}$, we find no significant difference in chaos at the higher 2009 incident angle.
\end{abstract}

Key words: comets: individual (Shoemaker-Levy 9) - hydrodynamics - methods: numerical

Online-only material: color figures

\section{INTRODUCTION}

Between 1994 July 16 and 22, fragments of the comet D/Shoemaker-Levy 9 (hereafter SL9) penetrated the Jovian atmosphere. This predicted impact gained great attention, with many Earth- and space-based telescopes aimed at this spectacle (see Harrington et al. 2004 for a review of the event). Despite impact occurrences of this nature being characterized as highly unlikely, another object collided with Jupiter sometime between UT 9 and 11 on 2009 July 19, drastically increasing the expected collision rate of $0.5-1.0 \mathrm{~km}$ bodies with Jupiter (SánchezLavega et al. 2010). Unfortunately, the 2009 impact itself was not directly observed; however, it was analyzed through observations of the impact's aftermath and was compared to the SL9 impacts (de Pater et al. 2010; Fletcher et al. 2010; Hammel et al. 2010; Orton et al. 2011; Sánchez-Lavega et al. 2010). Without a direct observation of the event, we use numerical simulations to seek a better understanding of the possible impact circumstances that could have produced this large atmospheric disturbance.

We use the ZEUS-MP 2 hydrodynamics code to simulate the collisions of several different types of impactors, sampling the impact parameter space constrained by observations. From simulation results, we garner information about possible penetration depths, plume development dynamics, and impact energies of potential 2009 impact scenarios. We also compare our results to numerical simulations of the SL9 impact event conducted by Korycansky et al. (2006) and Palotai et al. (2011). We compare energy deposition, penetration depth, and plume development between the SL9 and 2009 simulations.

This paper is organized as follows. Section 2 gives a description of our numerical model. The results of the simulations are given in Section 3. Section 4 discusses the results, compares them to observations, and also compares them to those of SL9 impact simulations. Lastly, the conclusions are contained in Section 5.

\section{IMPACT MODEL}

As with Korycansky et al. (2006) and Palotai et al. (2011), we used ZEUS-MP 2-a three-dimensional, parallel hydrodynamics code-for impact simulations. See Hayes et al. (2006) for ZEUS-MP 2 code details, and see Korycansky et al. (2002) and Korycansky \& Zahnle (2003) for the modifications made to the ZEUS-MP code to include multiple materials. By extending both the simulation time and the spatial extent of the computational grid in which the simulations are run, our ZEUS-MP-based code can model both the impact phase and the entry-response/blowout phase of the impact (here, we follow the terminology of Harrington et al. 2004). Hence, the impactor's entry into the atmosphere, impactor breakup, and plume formation and development are simulated in each run.

In the present simulations, we use the same coordinate system and a similar computational grid as Palotai et al. (2011). A Cartesian coordinate system is used in the simulations: $x_{1}$, the "along-track coordinate," is aligned with the impactor's initial trajectory; $x_{3}$, the "cross-track coordinate," is perpendicular to the impactor's initial trajectory; and $x_{2}$, the horizontal axis, is perpendicular to both $x_{1}$ and $x_{3}$. The local Cartesian coordinates for the Jovian reference frame are given by $x, y$, and $z$ and are related to $x_{1}, x_{2}$, and $x_{3}$ by the following equations, given by Korycansky et al. (2006):

$$
\begin{aligned}
& x=x_{2}, \\
& y=-x_{1} \sin \theta+x_{3} \cos \theta, \\
& z=x_{1} \cos \theta+x_{3} \sin \theta,
\end{aligned}
$$

where $\theta$ is the angle of incidence, i.e., the angle between $x_{1}$ and the local vertical, $z$. The origins of both coordinate systems coincide with the location of the 1 bar pressure level in the simulated Jovian atmosphere. Within and in close proximity to the impactor, the resolution is constant at 16 grid cells across the radius of the impactor (R16). The grid spacing then increases 
geometrically in each direction away from the R16 area. The resolution is again held constant $(4 \mathrm{~km}$ per grid cell) at the tail end of the grid, the area in which the impact plume develops and evolves.

As described by Korycansky et al. (2006), we use the Tillotson equation of state (EOS). The Tillotson EOS was derived for cases requiring high-velocity impact calculations, can describe the transition of shocked material into the vapor phase, but cannot represent a two-phase region, i.e., where a liquid and gas co-exist (Melosh 1989). The Tillotson EOS parameters used for the basalt and ice impactors are the same as those listed in Table 1 of Korycansky et al. (2006). These EOS parameters characterize the behavior of the different impactor materials.

For possible 2009 bolides, Sánchez-Lavega et al. (2010) suggest ice impactor diameters of $\sim 0.5 \mathrm{~km}$ up to $\sim 1 \mathrm{~km}$ based on comparisons to SL9 models and ablation rate considerations at higher angles of incidence. Hammel et al. (2010) suggest ice impactor diameters of 500-700 m based on similarities of the 2009 impact site to the E and R impact sites of SL9. There is observational evidence suggesting the possibility that the 2009 impactor was asteroidal in origin, rather than cometary (Fletcher et al. 2010; Hammel et al. 2010; Orton et al. 2011). From thermal heating and mass transport estimates, Orton et al. (2011) suggest diameters of 200-500 $\mathrm{m}$ for basalt impactors of density $2.5 \mathrm{~g} \mathrm{~cm}^{-3}$ (an impactor mass range of $\sim 1.05 \times 10^{13} \mathrm{~g}$ to $\sim 1.64 \times 10^{14} \mathrm{~g}$ ). The following eight impact cases were run to $\sim 30$ s after impact: $0.5 \mathrm{~km}$ and $1 \mathrm{~km}$ porous $\left(\rho=1.760 \mathrm{~g} \mathrm{~cm}^{-3}\right)$ and non-porous $\left(\rho=2.700 \mathrm{~g} \mathrm{~cm}^{-3}\right)$ basalt impactors, and $0.5 \mathrm{~km}$ and $1 \mathrm{~km}$ porous $\left(\rho=0.600 \mathrm{~g} \mathrm{~cm}^{-3}\right)$ and non-porous $\left(\rho=0.917 \mathrm{~g} \mathrm{~cm}^{-3}\right)$ ice impactors. We model $0.5 \mathrm{~km}$ bodies in order to sample relatively smaller impactors that satisfy size estimates for both ice and basalt bolides, and we model $1 \mathrm{~km}$ diameter impactors for easy comparison to previous SL9 models. An incident angle of $69^{\circ}$ from the vertical and an impact latitude of $55.10 \mathrm{~S}$ were used in the simulations (SánchezLavega et al. 2010). The gravitational acceleration at this latitude, including the $\mathrm{J} 2$ and centrifugal terms, is $2582 \mathrm{~cm} \mathrm{~s}^{-2}$. An impact velocity of $v=61.4 \mathrm{~km} \mathrm{~s}^{-1}$ was used for the purpose of comparison to previous SL9 simulations (Korycansky et al. 2006; Palotai et al. 2011). Several runs were also conducted to test the degree of dynamical chaos, the sensitivity of results to initial conditions, present in simulations with 2009 impact parameters (Korycansky et al. 2006).

\section{RESULTS}

Impact parameters for all the major cases run in the present paper, plus the parameters used by Palotai et al. (2011) for the SL9 case, are given in Table 1 , and a summary of simulation results is given in Table 2. A case label is given to each simulation. Figure 1 gives a series of snapshots of the $1 \mathrm{~km}$ non-porous ice impactor's simulated decent into the Jovian atmosphere. This figure shows the typical progression of our simulations. The first row of panels, $t=4.00 \mathrm{~s}$ after impact, shows the impactor traveling before it has begun to fall apart. As shown by Palotai et al. (2011), all of the impactor material is contained within a narrow trail following the body, constrained by the shock system on the trailing edge of the impactor. In the second row, $t=8.00 \mathrm{~s}$, the body has begun significant breakup and decelerates. The impactor material near and around the body begins to spread out quickly, and the shock system becomes turbulent. Material left behind by the bolide in the back half of the grid, within the high-temperature, low-density region that will form the plume, spreads out less rapidly. By $t=12.00 \mathrm{~s}$, the
Table 1

Impact Parameters

\begin{tabular}{lccccc}
\hline \hline $\begin{array}{l}\text { Case } \\
\text { Label }\end{array}$ & Material & $\begin{array}{c}\text { Density } \\
\left(\mathrm{g} \mathrm{cm}^{-3}\right)\end{array}$ & $\begin{array}{c}\text { Diameter } \\
(\mathrm{km})\end{array}$ & Angle & Latitude \\
\hline I05p & Ice & 0.600 & 0.5 & $69^{\circ}$ & $55^{\circ} .10 \mathrm{~S}$ \\
I05n & Ice & 0.917 & 0.5 & $69^{\circ}$ & $55^{\circ} .10 \mathrm{~S}$ \\
B05p & Basalt & 1.760 & 0.5 & $69^{\circ}$ & $55^{\circ} 10 \mathrm{~S}$ \\
B05n & Basalt & 2.700 & 0.5 & $69^{\circ}$ & $55^{\circ} .10 \mathrm{~S}$ \\
I10p & Ice & 0.600 & 1.0 & $69^{\circ}$ & $55^{\circ} .10 \mathrm{~S}$ \\
I10n & Ice & 0.917 & 1.0 & $69^{\circ}$ & $55^{\circ} 10 \mathrm{~S}$ \\
B10p & Basalt & 1.760 & 1.0 & $69^{\circ}$ & $55.10 \mathrm{~S}$ \\
B10n & Basalt & 2.700 & 1.0 & $69^{\circ}$ & $55^{\circ} .10 \mathrm{~S}$ \\
SL9p & Ice & 0.600 & 1.0 & $43^{\circ} .09$ & $44.02 \mathrm{~S}$ \\
SL9n & Ice & 0.917 & 1.0 & 43.09 & $44.02 \mathrm{~S}$ \\
\hline
\end{tabular}

Note. ${ }^{a}$ SL9 parameters used in Palotai et al. (2011).

impactor has become very incoherent and has almost reached its terminal depth. Impactor material and Jovian air in the back region begin their rapid ascent in the atmosphere, signifying the start of plume genesis. The plume is indicated by the growing red region. The majority of the impactor material travels to its terminal depth between $t=16.00$ and $24.00 \mathrm{~s}$, and the plume can be seen speeding up and rising straight up in the $x_{3}$-direction. As the impactor travels deeper, its vapor diffuses into the Jovian atmosphere, and the impactor fraction decreases with time. The plume increases its blowout speed and rises well above the initial impact trajectory of $x_{3}=0$. The impactor material in this region also continues to dissipate as the plume travels in the atmosphere. In general, all the impact simulations presented in this paper proceed as described above and resemble that pictured in Figure 1. Noteworthy differences in the depths reached by the impactors and the development of each plume do exist, however, and are explained in the following subsections.

\subsection{Terminal Depth and Energy Deposition}

Figure 2 shows the kinetic energy deposition curves for the porous and non-porous 2009 impact cases. Also included for comparison on each plot is the energy deposition curve of the appropriate SL9 impact case. These plots, similar to those of Korycansky et al. (2006), show the amount of energy an impactor releases to the surrounding atmosphere per unit of altitude traveled. The location of the initial and sudden increase of an energy deposition curve is an indicator of the height at which rapid and extensive structure loss of the impactor begins. Maximum energy deposition occurs when the nucleus of the impactor loses all coherency, and the impactor explodes.

The altitude at which the energy deposition returns to a value of zero is the terminal depth of the impactor. Each of the simulated impactors begins significant breakup at different altitudes and reach varying terminal depths. These terminal depths are listed in Table 2 and are given in the local vertical coordinate, $z$. A positive $z$ represents an altitude above the 1 bar level in the Jovian atmosphere, and a negative $z$ represents an altitude below the 1 bar level. The terminal depths are consistent with energy considerations: larger, more dense impactors will penetrate the deepest, whereas smaller, less dense impactors will reach shallower depths. The total energies of the impactors, obtained by integrating $d E / d z$, are given in Table 2 .

\subsection{Plume Development and "Pinch-off" Regions}

Figure 3 shows the plume structure at comparable times of plume evolution for each porous 2009 impact simulation. 

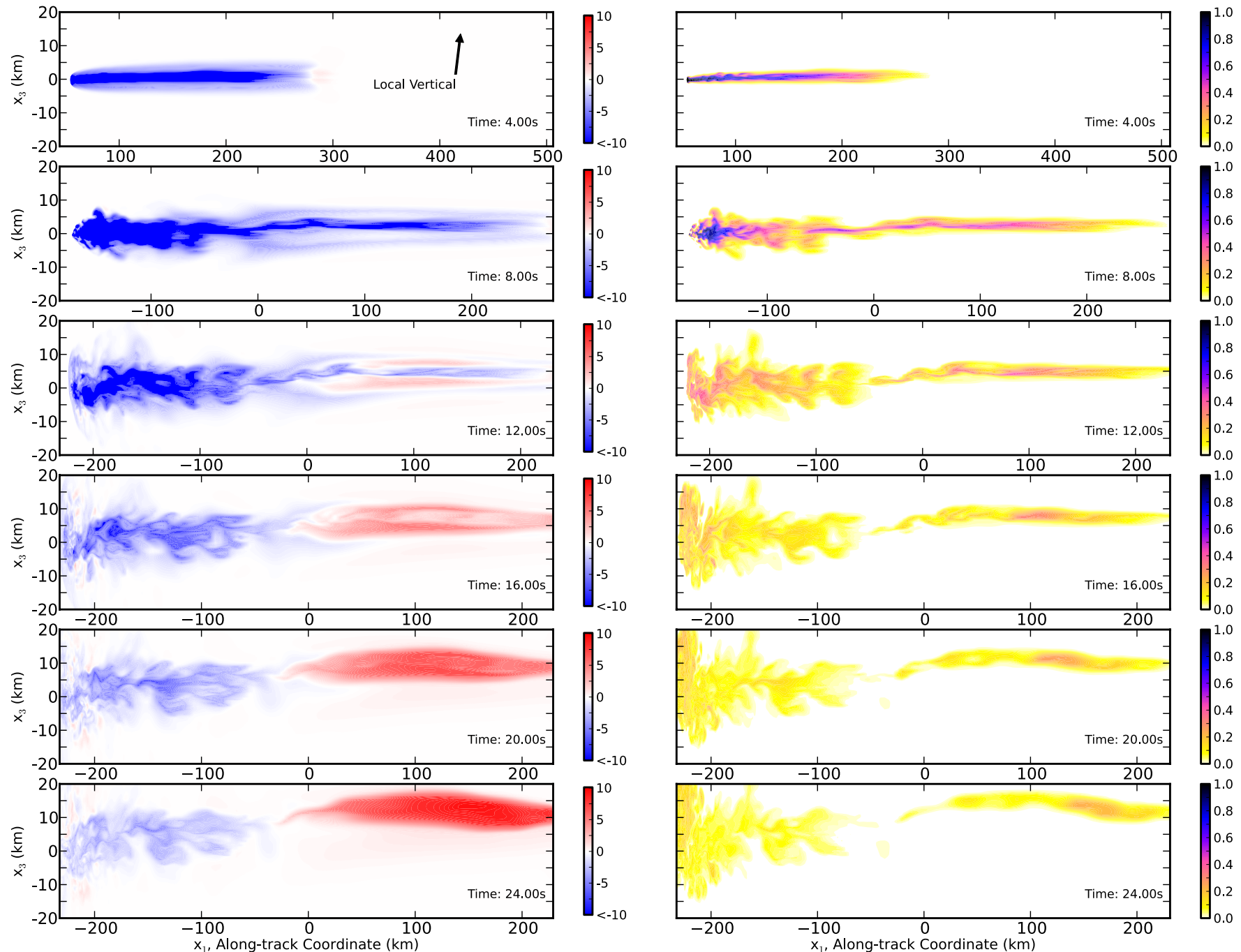

Figure 1. Progression of our impact simulations. Left: along-track velocity values $\left(\mathrm{km} \mathrm{s}^{-1}\right)$, i.e., the velocity in the $x_{1}$-direction. A red color indicates an upward velocity, material moving higher into the Jovian atmosphere; a blue color indicates a downward velocity, material moving deeper into the atmosphere. The red areas are indicators of the rising plumes. Right: the fraction of impactor material compared to Jovian air. The along-track coordinates are given at the bottom of the plots. The I10n case is shown here. The first panel shows an arrow indicating the direction of up in local Jovian coordinates. Note: the $x_{1}$ and $x_{3}$ length scales are not the same

(A color version of this figure is available in the online journal.)

Table 2

Simulation Results

\begin{tabular}{lcccccc}
\hline \hline $\begin{array}{l}\text { Case } \\
\text { Label }\end{array}$ & $\begin{array}{c}\text { Terminal Depth } \\
\text { a }\end{array}$ & $\begin{array}{c}\text { Pressure at } \\
\text { Terminal Depth } \\
(\mathrm{bar})\end{array}$ & $\begin{array}{c}\text { Total Energy } \\
(\mathrm{erg})\end{array}$ & $\begin{array}{c}\text { Maximum Plume Velocity } \\
\left(\mathrm{km} \mathrm{s}^{-1}\right)\end{array}$ & $\begin{array}{c}\text { Pinch-off Location } \\
\text { a }\end{array}$ & $\begin{array}{c}\text { Pressure at } \\
\text { Pinch-off Location } \\
(\mathrm{bar})\end{array}$ \\
\hline I05p & -24 & 2.44 & $7.4 \times 10^{26}$ & 7.5 & 21 & 0.365 \\
I05n & -36 & 3.52 & $1.1 \times 10^{27}$ & 9.1 & 19 & 0.408 \\
B05p & -54 & 5.67 & $2.2 \times 10^{27}$ & 8.0 & -1 & 1.06 \\
B05n & -55 & 5.83 & $3.3 \times 10^{27}$ & 8.3 & -2 & 1.10 \\
I10p & -61 & 6.72 & $5.9 \times 10^{27}$ & 10.3 & 4 & 0.891 \\
I10n & -80 & 10.2 & $9.1 \times 10^{27}$ & 12.5 & -4 & 1.19 \\
B10p & -102 & 15.8 & $1.7 \times 10^{28}$ & 11.0 & -18 & 2.00 \\
B10n & -114 & 19.7 & $2.6 \times 10^{28}$ & 11.2 & -23 & 2.37 \\
SL9p & -124 & 23.5 & $5.9 \times 10^{27}$ & 17.2 & -33 & 3.23 \\
SL9n & -150 & 35.0 & $9.1 \times 10^{27}$ & 16.0 & -50 & 5.15
\end{tabular}

Notes.

${ }^{a}$ Given in $z$, altitude relative to the 1 bar pressure level.

${ }^{\mathrm{b}}$ Results from simulation using parameters from Palotai et al. (2011). 

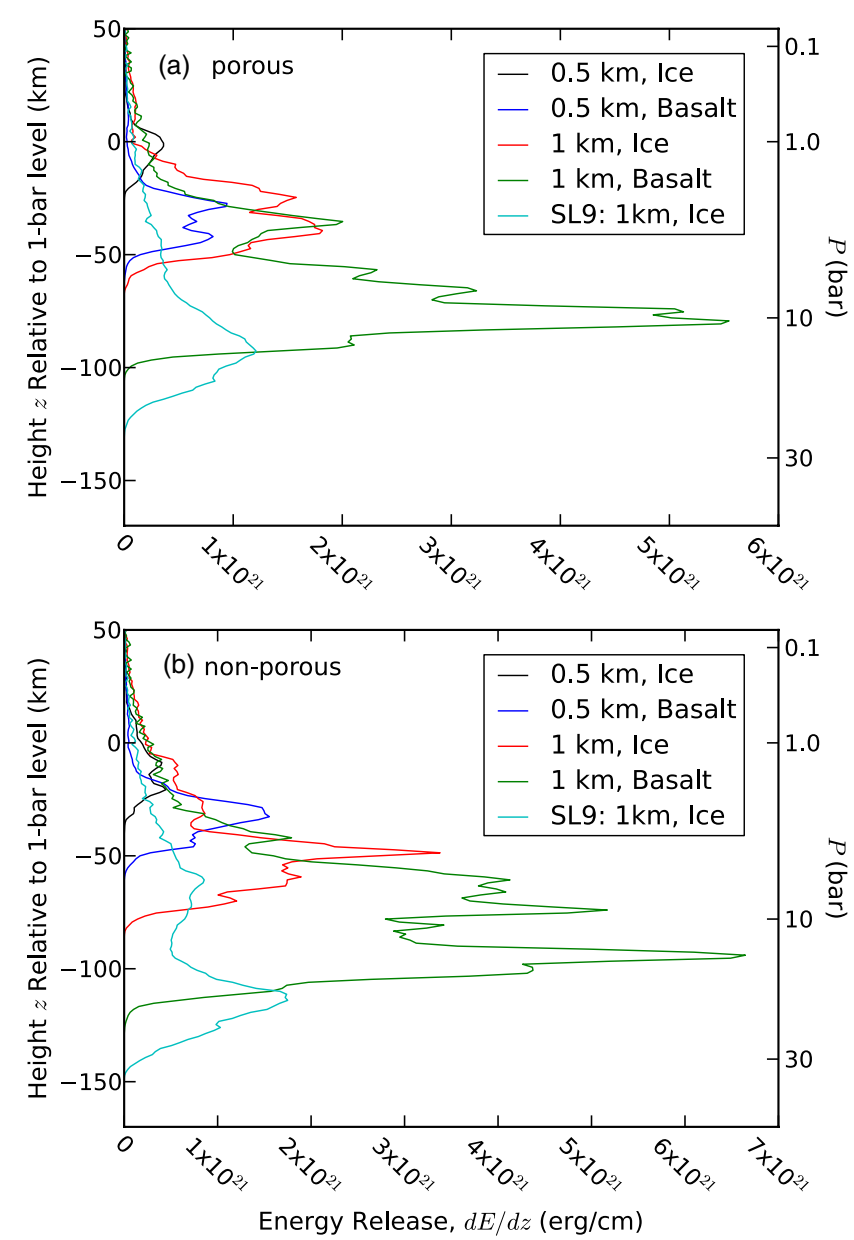

Figure 2. Kinetic energy deposition curves for the porous impact simulations (a) and the non-porous impact simulation (b). This plot demonstrates the increase of both terminal depth and total energy deposited as the size and density of the impactors grow.

(A color version of this figure is available in the online journal.)
Figure 4 shows the same for the non-porous 2009 impact simulations. Within the simulated $\sim 30 \mathrm{~s}$, higher resultant ejection speeds are reached in the $1 \mathrm{~km}$ diameter cases. Markedly smaller plumes and generally lower ejection speeds are seen in the $0.5 \mathrm{~km}$ impactor cases.

Figure 5 shows the velocity distribution of a rising plume and gives the fraction of impactor material contained within. All of the plume ejecta are above the initial impact trajectory. This is different from the SL9 simulations, where the plume expands to a greater diameter in the simulated time and still crosses the initial impact path (Palotai et al. 2011). The distribution of impactor material within the plume is similar to the SL9 simulation conducted by Palotai et al. (2011), however. A maximum of $\sim 10 \%$ impactor material is located near the top of the ejecting plume, and this fraction decreases as one moves deeper down the length of the plume. The ejection angles of the plumes are similar across all the 2009 cases, and within the simulated $\sim 30 \mathrm{~s}$, the plumes attain ejection angles of $60^{\circ}-70^{\circ}$ from the vertical.

Just as in the SL9 simulations conducted by Palotai et al. (2011), a pinch-off region appears in each of the 2009 cases. These regions are shown in Figures 3 and 4. Above this pinchoff level, heated Jovian atmosphere containing a small fraction of impactor material rises and expands as a plume (shades of red in Figures 3 and 4); below this level, a majority of the impactor material continues downward (shades of blue in Figures 3 and 4) and will later rise more slowly and independently of the ejecting plume as a bubble-like region of impactor material. The approximate location of this pinch-off region for each case is listed in Table 2.

The altitudes at which the pinch-off regions occur appear to correspond with the altitudes at which the impactor begins rapid and extensive structure loss (Palotai et al. 2011). We are currently working to better characterize and garner additional details about the relationship between the pinch-off region and impactor breakup.
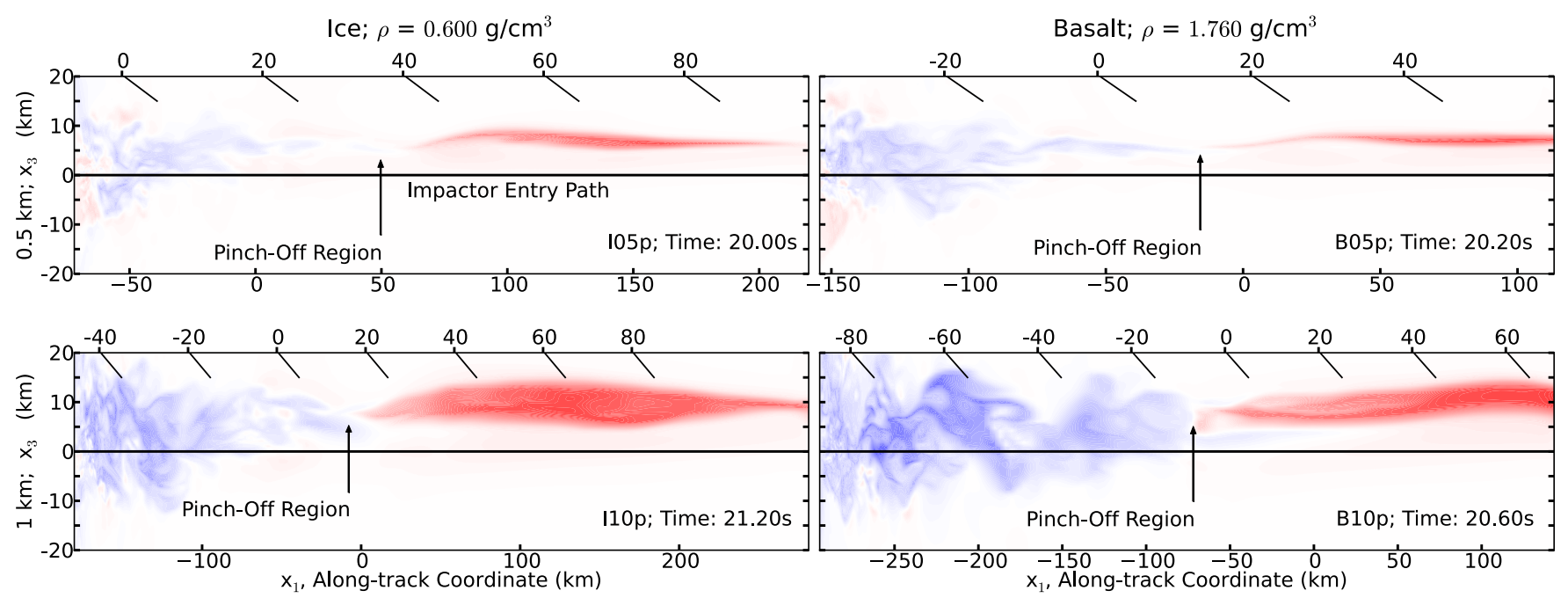

Figure 3. Plume structures of the porous 2009 impact cases. Along-track velocity is shown. The same color bar used for the left panels of Figure 1 also applies here. Top left: $0.5 \mathrm{~km}$, porous ice; top right: $0.5 \mathrm{~km}$, porous basalt; bottom left: $1 \mathrm{~km}$, porous ice; bottom right: $1 \mathrm{~km}$, porous basalt. The along-track coordinates are given at the bottom of the plots. The top of the plots indicates lines of constant height, $z(\mathrm{~km})$, in Jupiter's atmosphere. $z=0$ km represents the 1 bar pressure level. Note: the $x_{1}$ and $x_{3}$ length scales are not the same.

(A color version of this figure is available in the online journal.) 

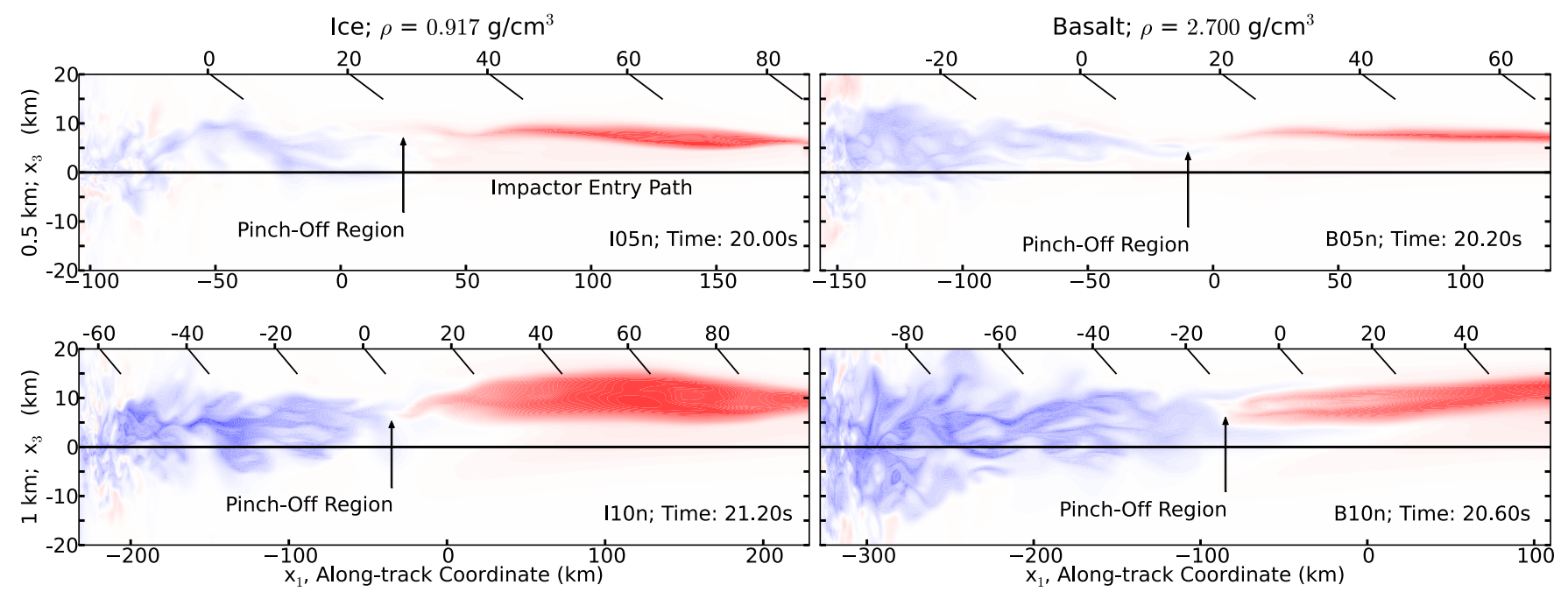

Figure 4. Plume structures of the non-porous 2009 impact cases. Along-track velocity is shown. The same color bar used for the left panels of Figure 1 also applies here. Top left: $0.5 \mathrm{~km}$, non-porous ice; top right: $0.5 \mathrm{~km}$, non-porous basalt; bottom left: $1 \mathrm{~km}$, non-porous ice; bottom-right: $1 \mathrm{~km}$, non-porous basalt. The along-track coordinates are given at the bottom of the plots. The top of the plots indicates lines of constant height, $z(\mathrm{~km})$, in Jupiter's atmosphere. $z=0 \mathrm{~km}$ represents the 1 bar pressure level. Note: the $x_{1}$ and $x_{3}$ length scales are not the same.

(A color version of this figure is available in the online journal.)

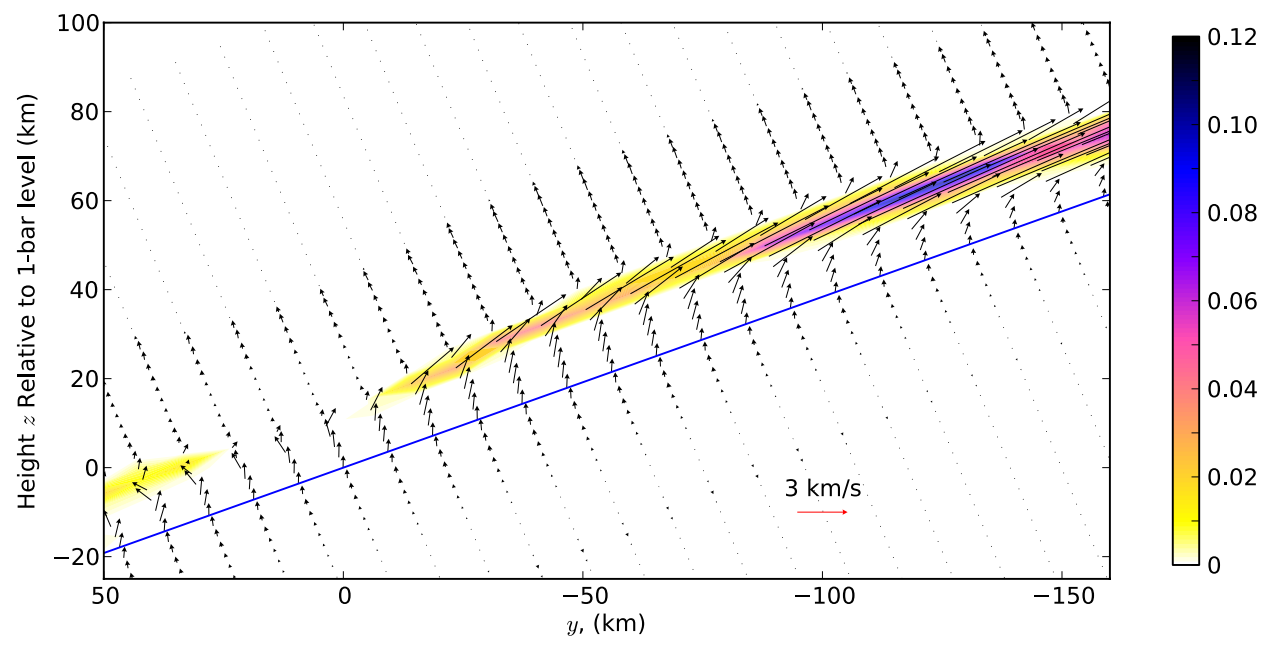

Figure 5. Velocity vector field of the impact plume for case I10p, $27.0 \mathrm{~s}$ after impact. Color gives the fraction of impactor material present in the rising plume. The straight, dark line indicates the initial trajectory of the impactor.

(A color version of this figure is available in the online journal.)

\section{DISCUSSION}

\subsection{Observations and Simulations}

Observations conducted by de Pater et al. (2010), Fletcher et al. (2010), Hammel et al. (2010), Orton et al. (2011), and Sánchez-Lavega et al. (2010) place several constraints on the impact parameters and the dynamics of the 2009 impact. In this section, we will examine these constraints and compare them to the results of the present simulations.

Two interesting constraints include the extent of debris distribution and temperature perturbations in the Jovian atmosphere as a result of the impact. Debris deposition was constrained roughly between the $10 \mathrm{mbar}$ and $300 \mathrm{mbar}$ levels (de Pater et al. 2010; Hammel et al. 2010; Orton et al. 2011). Thermal perturbations reached higher up in the atmosphere than the debris, up to about the 0.1-20 mbar levels, and were present slightly deeper into the atmosphere than the debris, down to the 400-600 mbar levels (de Pater et al. 2010; Orton et al. 2011). A lack of excess methane emission in the upper stratosphere is also an indicator of heating limited to pressures greater than 10 mbar (Fletcher et al. 2010). This is very different from the SL9 impacts, in which plumes reached thousands of kilometers above the 100 mbar level in Jupiter's atmosphere (Hammel et al. 1995; Jessup et al. 2000).

Enhanced levels of ammonia in the lower stratosphere also place boundaries on the penetration depth of the plume. Orton et al. (2011) found that a vertical profile of ammonia peaking in the 20-30 mbar region is required to reproduce the spectral shape of the $2009 \mathrm{NH}_{3}$ emission at the impact location. This enhanced presence of ammonia in the Jovian stratosphere implies the 2009 impact wake probably reached down to the 600-700 mbar levels, retrieving tropospheric ammonia and transporting it to the stratosphere as the plume rose (de Pater et al. 2010; Orton et al. 2011). However, it is likely that the downward jet that would form the plume did not penetrate much farther than the 700 mbar level since the $\mathrm{NH}_{3}$ gas was contained close to the center component of the impact streak (de Pater et al. 2010), and the jet probably did not make it to the Jovian water 


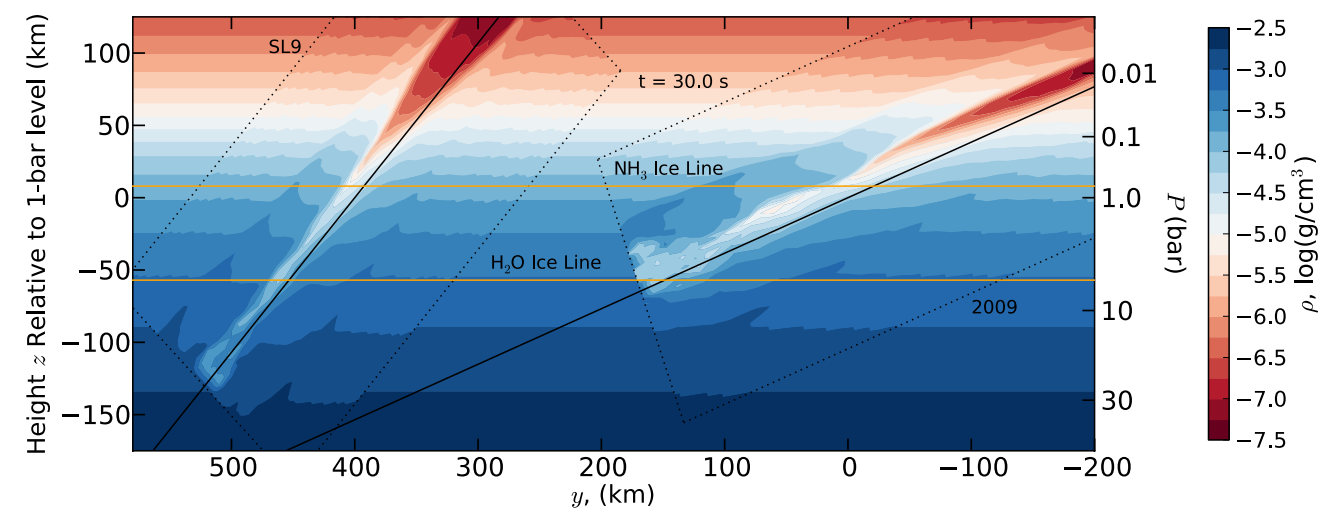

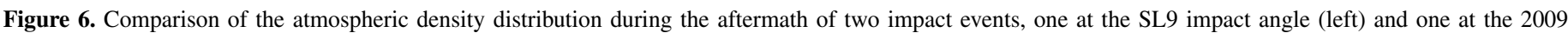

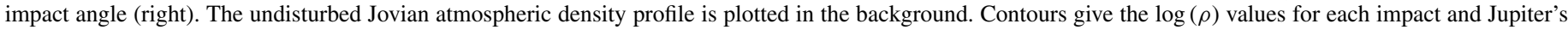

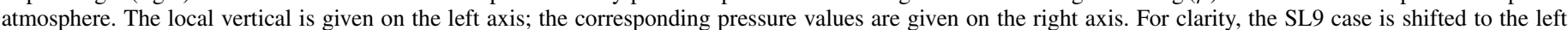

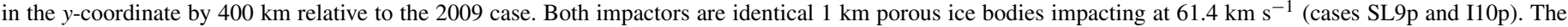
straight, dark lines indicate the initial trajectories of the impactors and the dotted lines indicate the boundaries of the simulations' computational grids.

(A color version of this figure is available in the online journal.)

cloud level since shock chemistry of the 2009 event favored production of ethane and other hydrocarbons over $\mathrm{CO}$ and $\mathrm{H}_{2} \mathrm{O}$ (Fletcher et al. 2010).

Table 2 gives the pressure levels at which the pinch-off regions occur for each of the conducted simulations. The pinch-off region is a measure of the deepest penetration of the fireball that forms the plume and is the lower limit from which material may be dredged up from the Jovian depths. In general, the plume jet is small and weak at the pinch-off region, but grows in speed as one moves up the plume channel. Cases I05p and I05n have pinch-off regions located at $\sim 365 \mathrm{mbar}$ and $\sim 410 \mathrm{mbar}$, respectively. Ice impactors of these sizes, densities, and impact velocity result in plumes that do not penetrate down to the stratospheric ammonia reservoir. Cases B05p and B05n result in relatively small plumes whose pinch-off regions penetrate down to the $\sim 1.10$ bar level, past the top levels of Jupiter's $\mathrm{NH}_{3}$ clouds, and speeds in the lower plume jet around the 700 mbar level reach $\sim 1.8 \mathrm{~km} \mathrm{~s}^{-1}$ for B05p and $\sim 1.0 \mathrm{~km} \mathrm{~s}^{-1}$ for B05n. Cases I10p and I10n have pinch-off regions located at $\sim 890$ mbar and $\sim 1.2$ bar, respectively. For these two $1 \mathrm{~km}$ ice cases, speeds in the lower plume jet at $\sim 700$ mbar reach about $1.8 \mathrm{~km} \mathrm{~s}^{-1}$ for I10p and $2.8 \mathrm{~km} \mathrm{~s}^{-1}$ for I10n. Cases B10p and B10n result in larger plumes that penetrate well past $700 \mathrm{mbar}$, down to pressures around the 2 and 3 bar levels, respectively, but stay above the $\mathrm{H}_{2} \mathrm{O}$ cloud tops around 6 bar. At the $\sim 700$ mbar level, speeds in the lower plume jet reach about $4.6 \mathrm{~km} \mathrm{~s}^{-1}$ for B10p and about $5.6 \mathrm{~km} \mathrm{~s}^{-1}$ for B10n.

Cases I05p and I05n do not satisfy the observational constraints, so it is likely that bodies with these impact characteristics did not cause the atmospheric response seen on Jupiter in 2009 July, setting a lower limit on the possible size, density, and impact velocity of the bolide. The plumes of cases B05p, B05n, I10p, I10n, B10p, and B10n penetrate deep enough to reach the ammonia clouds in the Jovian atmosphere, and so these impactors remain possible 2009 candidates. Further constraining the possibilities of the 2009 impact will require continued exploration of the possible impact parameter space, detailed ammonia transport calculations, and extending simulations both spatially and temporally.

Orton et al. (2011) give $7 \pm 2 \times 10^{26} \mathrm{erg}$ as a lower estimate for the energy of the impact. This is a lower estimate because it does not take into account the 4 days of cooling that passed between the impact and the observations nor the large amounts of energy lost to other dynamical processes in the impact, such as plume formation and the transport of atmospheric waves. All of the present simulations satisfy this lower limit.

\subsection{9 versus SL9 Impacts}

Figure 6 shows a snapshot of the aftermath of both an SL9 impact and a 2009 impact. The figure gives the density distribution for both cases, plotted against the undisturbed Jovian density distribution. Case SL9p and case I10p are used in the figure, i.e., the impacts in this figure are identical, except for the incident angle and the latitude at which the impactor strikes Jupiter. Both cases exhibit features characteristic of all our present impact simulations. At the terminal depths of the impactors, a relatively low-density region containing most of the impactor material exists. This is the bubble-like region below the pinch-off level seen in the simulations of Palotai et al. (2011). Shocks can be seen propagating away from the impact path, and at the boundaries of plume formation, two shock waves can be seen. The lowest-density region marks the location of the plume. In the upper atmosphere, the plume gains speed, rises, and expands; in the lower atmosphere, the plume becomes slender, tapering to the pinch-off region. Though both impactors are identical and share general characteristics, the terminal depths and plume development are strikingly different at the differing incident angles.

In this particular comparison, the terminal depth of the SL9 impactor is $\sim 60 \mathrm{~km}$ deeper than the 2009 impactor's terminal depth. This is mainly due to the larger incident angle of the 2009 impact: for a given distance traveled along the path of each impactor (in $x_{1}$ ), displacement in height $z$ will be smaller at higher incident angles. The pinch-off regions differ by a little more than a scale height, with the SL9 pinch-off reaching to $\sim 30 \mathrm{~km}$ below the 1 bar level and the 2009 pinch-off occurring $\sim 4 \mathrm{~km}$ above the 1 bar level. There is also a distinct difference between the developing plumes. For the SL9 case, the plume begins to expand significantly as it rises in the atmosphere. The SL9 plume reaches a diameter of $\sim 35 \mathrm{~km}$ by $30 \mathrm{~s}$ after impact. Such rapid expansion is not seen in the 2009 plume, however. During the formation of the 2009 plume, it rises above the impact path while undergoing relatively little expansion. By $30 \mathrm{~s}$ after impact, the 2009 plume only reaches a diameter of $\sim 15 \mathrm{~km}$. The difference in plume sizes can be seen in Figure 6 which shows the SL9 plume about twice the size of the 2009 plume. Table 2 

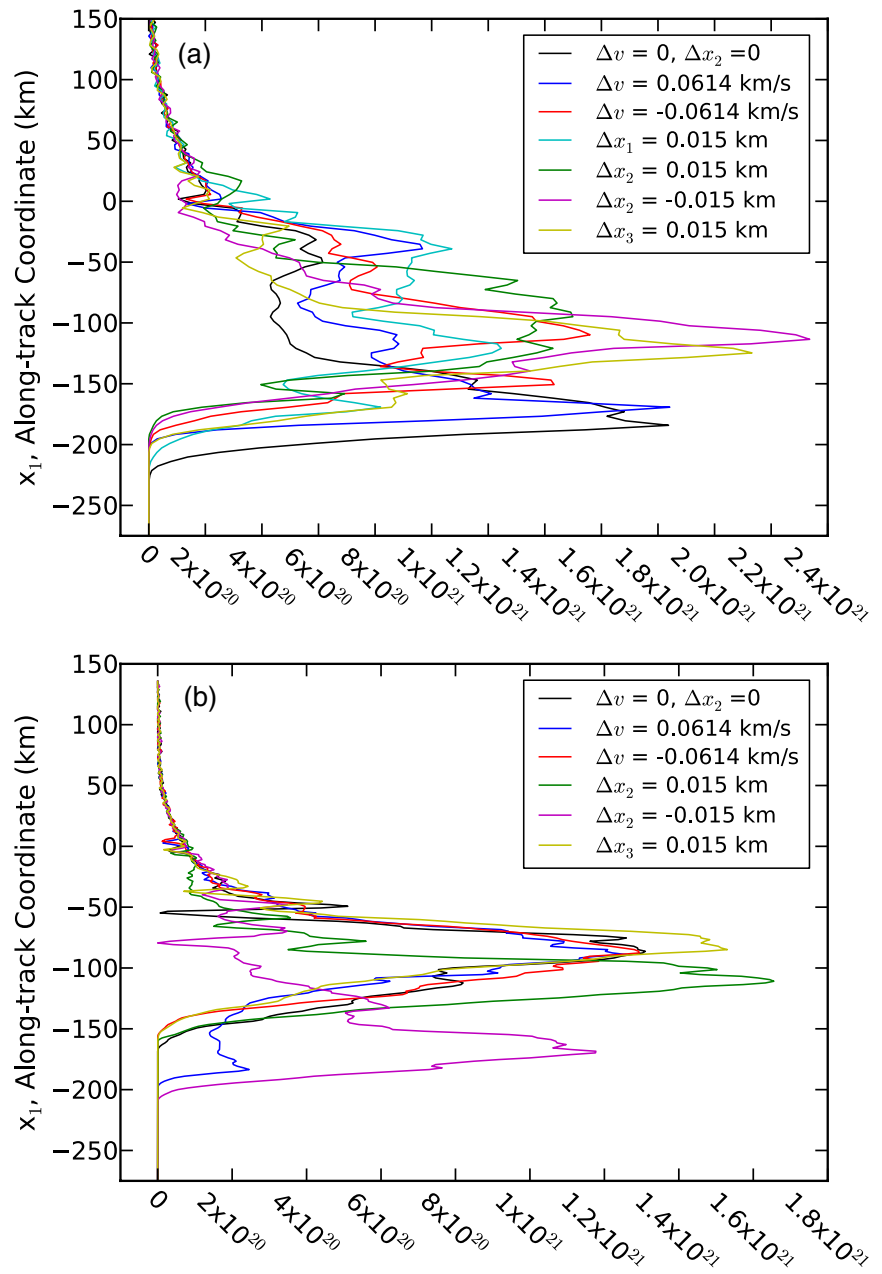

Energy Release, $d E / d z$ (erg/cm)

Figure 7. Dynamical chaos analysis. Kinetic energy deposition curves at the 2009 incident angle (a) and the SL9 incident angle (b). The black line gives the energy deposition for the nominal case of a $1 \mathrm{~km}$ porous ice impactor traveling at $v=61.4 \mathrm{~km} \mathrm{~s}^{-1}$. The bottom plot is Figure 7(d) from Korycansky et al. (2006).

(A color version of this figure is available in the online journal.)

reveals that the SL9 plume contains air and entrained impactor material traveling at speeds about $7 \mathrm{~km} \mathrm{~s}^{-1}$ greater compared to the 2009 plume. The larger plume and heightened speeds in the SL9 case relative to that of 2009 implies a dependance of plume size and maximum plume height on incident angle.

\subsection{Dynamical Chaos}

In order to observe the sensitivity of the present results to initial conditions, we conducted several $1 \mathrm{~km}$ porous ice impactor simulations with slightly different initial conditions compared to a nominal case. These differences in initial conditions include a change in the impact velocity $(\Delta v)$ by $0.1 \%$ and a shift in the initial position of the impactors (in each direction $x_{1}, x_{2}$, and $x_{3}$ ) by half a grid cell, $\sim 15 \mathrm{~m}$ in this case. The variation in terminal depths and peak energy deposition locations is a measure of the dynamical chaos present in the simulations. Korycansky et al. (2006) conducted this same analysis and found that among the four SL9 impact cases they simulated, a $1 \mathrm{~km}$ porous ice impactor was subject to the highest degree of dynamical chaos. Only slightly changing initial conditions for this case resulted in relatively large variations in the terminal depths and peak energy deposition locations.

Figure 7(a) gives our chaos analysis of the 2009 impact for the porous ice case. Comparing Figure 7 to Korycansky et al.'s (2006) Figure 7(d), seen here as our Figure 7(b), we do not find the dynamical chaos to be significantly different at an incident angle of $\theta=69^{\circ}$. The standard deviation of the terminal depths at the 2009 impact angle is $S_{i 09} \approx 13.2 \mathrm{~km}$ in the along-track coordinate, and the range in terminal depths is about $44 \mathrm{~km}$. At the $\theta \approx 45^{\circ}$ SL9 angle of incidence, the standard deviation of the terminal depths is $S_{\mathrm{SL} 9} \approx 20.8 \mathrm{~km}$ in the along-track coordinate, and the range in terminal depths is about $53 \mathrm{~km}$.

We conducted a simple $F$-test to compare the variation in terminal depths between the 2009 and SL9 cases. Our test $F$ statistic, $F=S_{\mathrm{SL} 9}^{2} / S_{i 09}^{2}=2.48$, was compared to the critical $F$ statistic at the 5\% level, $F=4.39$. Though visually the terminal depths for the 2009 porous ice case seem less scattered, and the range and standard deviation of these terminal depths are less at the 2009 incident angle, we cannot say that the standard deviation of the terminal depths at the SL9 incident angle is significantly larger than at the 2009 incident angle because our test $F$ statistic was not greater than the critical value.

\section{CONCLUSIONS}

We have presented several possibilities for the impact and immediate aftermath of the 2009 collision into the Jovian atmosphere. The results of the present simulations provide insight into the impact event and also provide information about the variation in atmospheric response due to changes in impact parameters. At the estimated 2009 incident angle of $\theta=69^{\circ}$, we see several differences between plumes generated from $0.5 \mathrm{~km}$ impactors and $1 \mathrm{~km}$ impactors. Within the simulated $\sim 30 \mathrm{~s}$, the $0.5 \mathrm{~km}$ impactor events produce relatively smaller and slower plumes while the $1 \mathrm{~km}$ impactor events produce relatively larger and faster plumes.

The penetration depths of the impactors and the pinch-off regions are associated with the nature of the impactors: at a given incident angle, the larger the impactor and the heavier the impactor material, the deeper the locations of the terminal depth and the pinch-off region. Dynamical chaos present at the 2009 incident angle for the $1 \mathrm{~km}$ porous ice impactor did not prove to be significantly less than that of the most chaotic impactor case given by Korycansky et al. (2006).

Comparing the aftermaths of an SL9 impact and the 2009 event reveals several differences that may have consequences for the observable manifestation of Jupiter's atmospheric response. The impact plume produced at the SL9 incident angle is significantly larger and faster than that of the impact plume produced at the 2009 incident angle.

Given observations of thermal perturbations, debris deposition, and ammonia transport in the Jovian atmosphere after the 2009 event, constraints have been placed on the possible outcomes of the impact, including plume speeds and pinchoff region locations (de Pater et al. 2010; Fletcher et al. 2010; Hammel et al. 2010; Orton et al. 2011). Of the eight cases considered in the present paper, the $0.5 \mathrm{~km}$ ice cases cannot explain the atmospheric disturbance observed after the 2009 impact; these impactors' plume jets do not penetrate deep enough to explain stratospheric $\mathrm{NH}_{3}$ observations (de Pater et al. 2010; Orton et al. 2011). We thus set a lower limit on the size and density of the 2009 impactor. All $1 \mathrm{~km}$ impactor plumes and the $0.5 \mathrm{~km}$ basalt impactor plumes reach down to the ammonia ice cloud level in Jupiter's troposphere. To better constrain the 
impact characteristics of the 2009 event, more simulations are required spanning more of the possible parameters proposed by observations. Ammonia transport from the upper troposphere to the stratosphere by means of the rising plume jets must also be looked at in more detail. Through this analysis, we will narrow the range of possible impactor circumstances that produced the atmospheric disturbance on Jupiter in 2009.

This work was supported by National Science Foundation Grants AST-0813194, AST-0964078, and AST-1109729 and NASA Planetary Atmospheres Program Grant NNX11AD87G.

\section{REFERENCES}

de Pater, I., Fletcher, L. N., Pérez-Hoyos, S., et al. 2010, Icarus, 210, 722

Fletcher, L. N., Orton, G. S., de Pater, I., \& Mousis, O. 2010, A\&A, 524, A46
Hammel, H. B., Beebe, R. F., Ingersoll, A. P., et al. 1995, Science, 267, 1288 Hammel, H. B., Wong, M. H., Clarke, J. T., et al. 2010, ApJ, 715, L150

Harrington, J., de Pater, I., Brecht, S. H., et al. 2004, in Lessons from ShoemakerLevy 9 about Jupiter and Planetary Impacts, ed. F. Bagenal, T. E. Dowling, \& W. B. McKinnon (New York: Cambridge Univ. Press), 159

Hayes, J. C., Norman, M. L., Fiedler, R. A., et al. 2006, ApJS, 165, 188

Jessup, K. L., Clarke, J. T., Ballester, G. E., \& Hammel, H. B. 2000, Icarus, 146, 19

Korycansky, D. G., Harrington, J., Deming, D., \& Kulick, M. E. 2006, ApJ, 646, 642

Korycansky, D. G., \& Zahnle, K. J. 2003, Icarus, 161, 244

Korycansky, D. G., Zahnle, K. J., \& Mac Low, M. 2002, Icarus, 157, 1

Melosh, H. J. (ed.) 1989, Impact Cratering: A Geologic Process (New York: Oxford Univ. Press)

Orton, G. S., Fletcher, L. N., Lisse, C. M., et al. 2011, Icarus, 211, 587

Palotai, C., Korycansky, D. G., Harrington, J., Rebeli, N., \& Gabriel, T. 2011, ApJ, 731, 3

Sánchez-Lavega, A., Wesley, A., Orton, G., et al. 2010, ApJ, 715, L155 